\title{
Pediatric Stomach and Duodenal Imaging
}

\author{
Pritviraj SK ${ }^{1}$ - Devasenathipathy Kandasamy ${ }^{1} \quad$ Manisha Jana ${ }^{1} \quad$ Raju Sharma ${ }^{1} \quad$ Arun Kumar Gupta ${ }^{1}$ \\ ${ }^{1}$ Department of Radiodiagnosis, All India Institute of Medical \\ Sciences, New Delhi, India \\ Address for correspondence Raju Sharma, MBBS,MD, MAMS, \\ FICR, Department of Radiodiagnosis, All India Institute of Medical \\ Sciences, New Delhi, India (e-mail: raju152@yahoo.com).
}

J Gastrointestinal Abdominal Radiol ISGAR 2021;4:94-108.
Abstract
Keywords
- pediatric
- gastrointestinal
- radiograph
- ultrasound
$-\mathrm{CT} / \mathrm{MRI}$

Pediatric stomach and duodenum abnormalities constitute a unique spectrum of conditions unlike adults which are categorized broadly into congenital, inflammatory, neoplastic and miscellaneous conditions. The aim of this article is to describe the role of imaging modalities in gastric and duodenal pathology in children. Some of the entities are unique to this age group and their imaging findings are also highlighted.

\section{Introduction}

A wide and unique spectrum of pathological entities can involve the stomach and duodenum in the pediatric patient. Broadly, they can be divided into congenital, inflammatory/infective, and neoplastic. Any disease process affecting the stomach or the duodenum tends to present with vomiting as the primary complaint. Establishing the diagnosis often requires a judicious multimodality approach, including radiograph, contrast study, ultrasound (US), computed tomography (CT), and magnetic resonance imaging (MRI). This article describes various imaging modalities, and their role in imaging of gastric and duodenal pathology in the pediatric patient. Some of the entities are unique to this age group and their imaging findings are also highlighted.

\section{Imaging Modalities}

\section{Radiograph}

Abdominal radiographs are still used as the first line of investigation in a number of pediatric gastric and duodenal pathologies such as gastric hernia, volvulus, congenital hypertrophic pyloric stenosis (CHPS), duodenal atresia, and gastric bezoar. The advantages of radiographs include low cost and easy availability in addition to providing a wealth of information. Commonly used radiographic projections are supine, erect, and decubitus; the latter two being especially useful for small pneumoperitoneum.

\section{Contrast Study}

Contrast-based fluoroscopic studies are useful in evaluating upper GI (gastrointestinal) pathology, and diluted barium is the preferred contrast agent for upper GI evaluation in children. Water-soluble iodinated contrast is preferred in cases where barium is either relatively or absolutely contraindicated. It is preferred in neonates and children and wherever leak or perforation is suspected. Low-osmolar iodinated contrast agents are preferred over high-osmolar contrast agents if there is an increased risk of aspiration, because of the risk of pulmonary edema with the latter. Barium swallow is used to evaluate the pathology in the upper GI tract starting from oropharynx up to the gastric fundus. Barium meal is the study used to evaluate mainly the stomach and proximal small bowel. Various pathologies like gastric hernia, volvulus, bezoar, CHPS and duodenal atresia and web can be well demonstrated in this contrast study. Pyloric stenosis is one of the most frequent causes of vomiting in young infants, which can be evaluated by barium meal examination, although nowadays it is almost completely replaced by US. Barium meal follow through remains the imaging reference standard for the diagnosis of malrotation with or without volvulus. The aim is to evaluate the entire small bowel for any abnormality in peristalsis and mucosa up to the ileocecal junction. published online

March 1, 2021
DOI https://doi.org/

$10.1055 / \mathrm{s}-0041-1723926$

ISSN 2581-9933.
(C) 2021. Indian Society of Gastrointestinal and Abdominal Radiology. This is an open access article published by Thieme under the terms of the Creative Commons Attribution-NonDerivative-NonCommercial-License, permitting copying and reproduction so long as the original work is given appropriate credit. Contents may not be used for commercial purposes, or adapted, remixed, transformed or built upon. (https://creativecommons.org/licenses/by-nc-nd/4.0/).

Thieme Medical and Scientific Publishers Pvt. Ltd. A-12, 2nd Floor, Sector 2, Noida-201301 UP, India 


\section{Ultrasound}

US examination in children is a simple and cost-effective investigation which does not have side-effects related to ionizing radiation. Real-time evaluation is another advantage of this modality. US has less importance in the evaluation of GI pathologies in comparison to cross-sectional examinations. Nevertheless, they have a niche role in certain situations and can yield better insight compared to cross-sectional studies. One of the conditions where US has better diagnostic utility over other modalities is evaluation of suspected duplication cyst. In addition, US is the imaging modality of choice for hypertrophic pyloric stenosis in infants. The addition of color Doppler can help evaluate vascular lesions such as hemangioma. Introduction of contrast-enhanced ultrasound (CEUS) has further enhanced its role in a variety of bowel lesions. The accuracy of CEUS in the evaluation of disease activity in Crohn's disease is found to be comparable or superior to MR enterography. ${ }^{1}$

\section{Computed Tomography}

$\mathrm{CT}$ is a problem-solving modality in pediatric gastric and duodenal pathology and is not used as the first-line investigation but is reserved for resolving diagnostic dilemmas. Issues that should be addressed in pediatric CT examination include psychological preparation of children and parents, the need for sedation or general anaesthesia, oral contrast material or/and intravenous (IV) contrast material preparation. The primary goal should be to achieve diagnostic image quality instead of optimal image quality in order to minimize the radiation dose to the child. Dose-reduction techniques are adopted to reduce the radiation dose while maintaining the diagnostic quality of the examination. The scan and technical parameters should be tailored to the size of the child, the body region of interest and the clinical question. There are few technological advances in CT technology in the last decade which reduces the radiation dose. The most important of them is the iterative reconstruction algorithm which can bring down the radiation by up to $90 \%$ while maintaining the image quality. ${ }^{2}$

NCCT acquisition is not always necessary. Dual-energy CT can create virtual non enhanced CT from the contrast CT. In CT enterography, bowel distension is a critical requirement for the evaluation of bowel wall, especially in inflammatory pathologies.

\section{Magnetic Resonance Imaging}

Abdominal MRI is being increasingly used in children, because it is safe from radiation side effects and also provides better soft-tissue resolution. Disadvantage is the need for sedation or general anesthesia.

There are currently many motion compensating techniques available with MRI which can address this problem. MR enterography is the modality of choice for inflammatory bowel disease both at baseline and follow-up. It is performed using $900 \mathrm{~mL}$ of oral contrast given over 45 to 60 minutes followed by MR acquisition. It provides multiple paradigms for characterization of bowel abnormalities.

\section{Nuclear Scintigraphy}

Scintigraphy assesses the function of the tissue by using radiotracers and its uptake at cellular levels with different techniques. Role of nuclear medicine in children is well established in renal and urinary tract pathologies. It also has a niche role in certain GI tract abnormalities like duplication cyst in which Tc99 labelled pertechnetate scan shows tracer uptake in the cyst if it contains ectopic gastric mucosa, confirming the diagnosis. The addition of single positron emission computed tomography (SPECT) over planar imaging improves the diagnostic accuracy but comes at a cost of increased radiation dose. ${ }^{3}$

\section{Congenital Entities}

\section{Hypertrophic Pyloric Stenosis}

This is the most common gastric pathology seen in infants. It usually presents between the second and sixth week of life, more commonly in the White population, in males (male: female ratio 4:1) and typically in first-born children. The etiology for the condition remains unknown.

Clinical presentation: typically presents with features of gastric outlet obstruction such as vomiting after feeding. It is the most common surgical cause of vomiting in infants. Late clinical manifestations include weight loss and visible gastric peristaltic activity with a palpable pyloric "olive."

\section{Imaging Features:}

Radiograph: Although radiograph is of limited utility, abdominal radiograph when performed may show gross gastric distension with no gas shadow distal to the stomach. ${ }^{4}$

US: This is the modality of choice for diagnosing this condition. On US, first the pylorus needs to be located, which is most commonly posterior and medial to the gallbladder.

If the single muscular wall thickness is more than $3 \mathrm{~mm}$, it is highly specific for the diagnosis. The other sonographic feature is the length of the pyloric canal, which is abnormal if it is more than $16 \mathrm{~mm}$. The hypertrophied pylorus may give an appearance similar to uterine cervix (cervix sign). ${ }^{5}$

Barium meal: Barium is the most commonly used oral contrast material, and it may be administered either by means of a nursing bottle or through a nasogastric tube. It is performed only if the findings on US are equivocal. Two important signs of CHPS are the "shoulder" sign in the antrum and the "shoulder" or "mushroom" sign in the base of the duodenal cap. These are produced by the protrusion of the muscle into the lumen of the antrum and into the duodenal cap, respectively ${ }^{6}$ (- Figs. 1 and $\mathbf{2}$ ).

\section{Pylorospasm}

Pylorospasm is an entity which can mimic hypertrophic pyloric stenosis in infants with similar clinical presentation of gastric outlet obstruction like vomiting. However, it needs to be differentiated from the hypertrophic pyloric stenosis, since the management of both the entities is completely different. CHPS is treated surgically with pyloroplasty and pylorospasm is usually treated conservatively.

Clinical presentation: Similar to the CHPS, infants with pylorospasm also present with complaints of vomiting. 
Hence, this entity should also be kept in mind in infants presenting with features of gastric outlet obstruction.

Radiograph: The abdominal radiograph AP view may just demonstrate the dilated stomach. Besides, these radiographs are not helpful in differentiating it from CHPS.
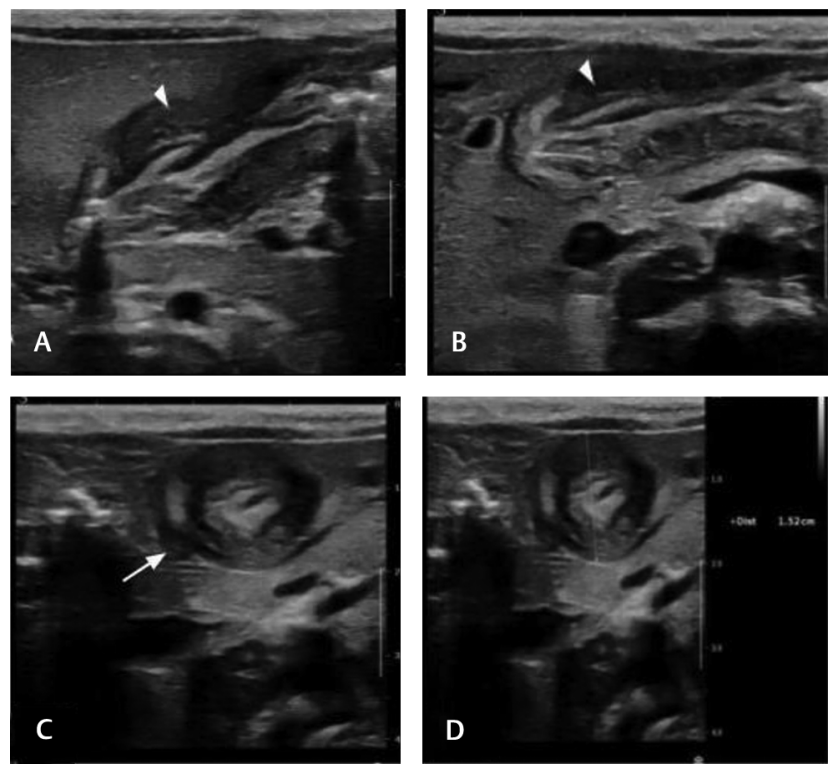

Fig. 1 Congenital hypertrophic pyloric stenosis. (A, B) Ultrasound (US) (longitudinal plane) shows gross thickening of the muscular layer (arrow head) of the pylorus with significant luminal narrowing. (C) US (transverse plane) shows echogenic mucosa in the central part, with surrounding thickened muscular layer showing target sign (arrow). (D) The diameter of the pylorus (serosa to serosa) in transverse plane measures $15 \mathrm{~mm}$.
US: is the modality of choice which has been shown to be of some help in differentiating it from CHPS. The pylorus measurements on US are usually abnormal, however they are lesser and not in the range of CHPS.

Barium meal: This can be helpful in showing the pyloric abnormality; however, it does not have a role in differentiating it from CHPS. ${ }^{7}$

\section{Congenital Pyloric Atresia/Membrane Spectrum}

Congenital pyloric atresia is a rare entity whose exact etiology is unknown. One of the theories proposes a possible arrest of development between 5th and 12th week of gestation, leading to failure of canalization of the pylorus. Pyloric atresia can be isolated or associated with epidermolysis bullosa and intestinal atresia.

Congenital pyloric atresia can be classified morphologically into three types:

Type 1: pyloric membrane (57\%), which may be multiple.

Type 2: pyloric atresia without a gap (34\%).

Type 3: pyloric atresia with a gap between the stomach and the duodenum (9\%)

Clinical features: Infants with pyloric atresia present with nonbilious vomiting after birth, feeding difficulty and, rarely, upper abdominal distension. Antenatally, these cases may be associated with polyhydramnios, and if there is any delay in the diagnosis, it may lead to complications such as pulmonary aspiration and perforation.

Radiograph: Abdominal radiograph may demonstrate the single bubble sign, which is due to the distended stomach. Distally bowel loops are gasless and collapsed.

US: Antenatally, it can be diagnosed using US findings of polyhydramnios and distended stomach
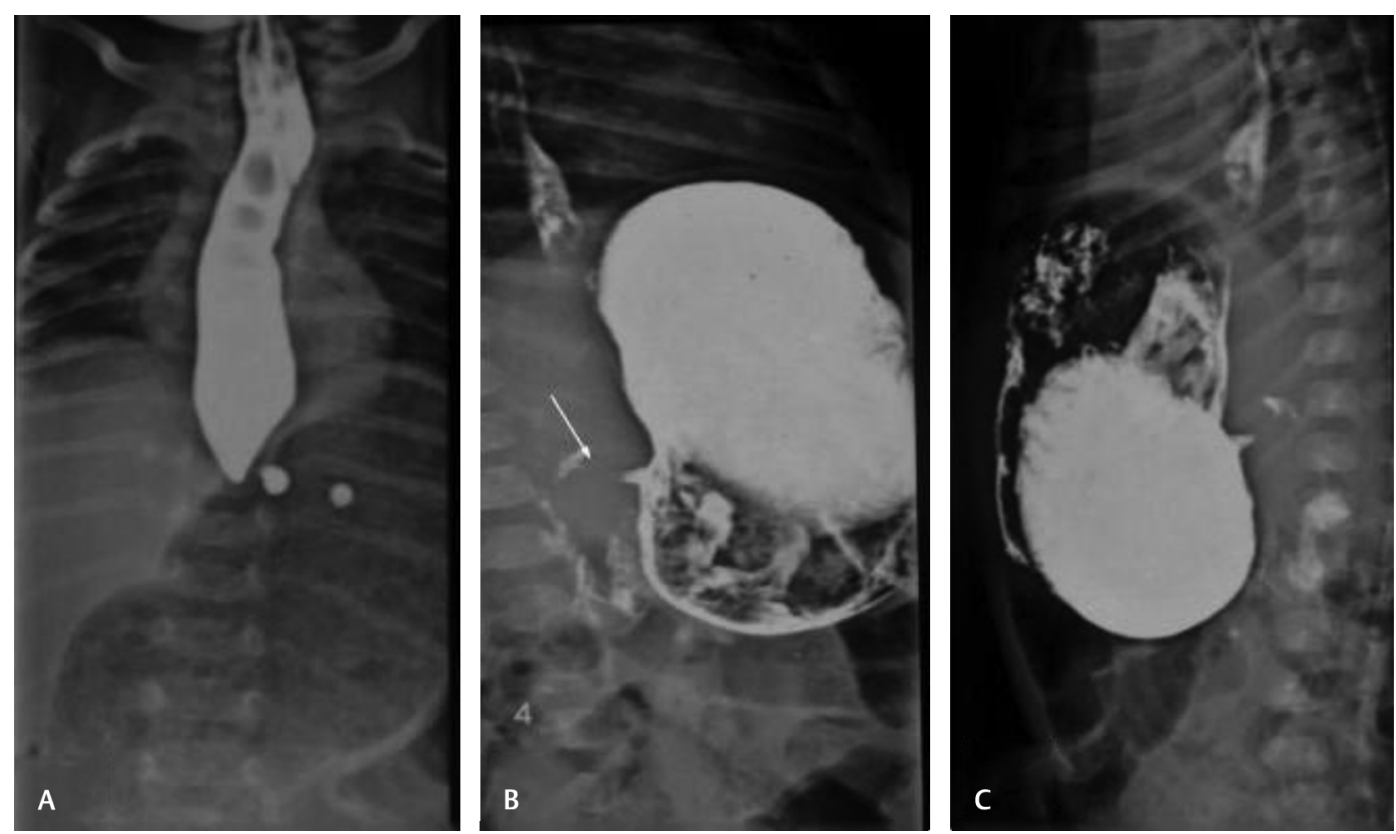

Fig. 2 Congenital hypertrophic pyloric stenosis. (A) Barium meal study in early phase (frontal view) shows normal contrast-filled esophagus with gas-filled distended stomach. (B, C) Images in later phase acquired in oblique and lateral views show distended contrast-filled stomach with elongated pylorus (white arrow) and narrow lumen (string sign). 
Barium study: Barium meal study is not necessary to diagnose the condition; however, if performed, it can show the obstruction, depending on the type. ${ }^{8}$

\section{Gastric Volvulus}

Volvulus involving the stomach is rare in children when compared to adults. Less than a third of all gastric volvulus occur in children. There are two types of gastric volvulus, based on the axis of rotation of the stomach. Organoaxial is the most common type occurring in adults. It occurs due to rotation along its long axis, where the greater curvature becomes cranial in position and lesser curvature becomes caudal in position, and it occurs in the setting of trauma or may be associated with a coexistent paraesophageal hernia. Mesenterico-axial is the common type of gastric volvulus in children, which occurs due to rotation along the short axis in which gastroesophageal (GE) junction is displaced caudally, whereas pylorus moves proximally.

The clinical symptoms associated with volvulus commonly are nonspecific and include pain, nausea, and vomiting.

Radiograph: Abdominal radiograph shows absence of normal gastric bubble with herniation of large part of stomach above the diaphragm with air fluid levels in children with hernia or eventration. It may also show a hugely distended stomach with a large air fluid level.

Barium study: Upper GI series done to evaluate the volvulus shows altered relation of lesser and greater curvature or cardia and pylorus, depending on the type of gastric volvulus.

CT: Contrast-enhanced CT is often performed in the setting of epigastric pain and vomiting and can help to confirm the rotation of the herniated stomach and the transition point in cases of gastric volvulus. The relationship of GE junction and the pylorus and associated herniation and complications are much better demonstrated on CT, especially on coronal reformatted images ${ }^{9}$ (-Fig. 3).

\section{Gastric Duplication Cyst}

Gastric duplication cyst is a rare anomaly; it comprises $4 \%$ of the total GI duplication cysts. Gastric duplication cysts present at variable age from infancy to adults. It is known to be more prevalent in females and divided into tubular and cystic types. Cystic type is the most common, accounting for up to $80 \%$ and usually not in communication with the gastric lumen. Tubular type is less common and can have communication with the gastric lumen. ${ }^{10}$

Clinical features: Presentation may vary from asymptomatic children detected incidentally to children with complaints of pain and discomfort in epigastrium, vomiting and blood in stool.

Radiograph: Abdominal radiograph has no role in the diagnosis and may be apparently normal in the majority of cases, except in children with large lesions where they may show mass effect.

Barium meal: Barium meal is not necessary for the diagnosis; however, if performed, it may show mass effect in the form of extrinsic compression over the stomach, which may help in suspecting the condition.

US: It is the most useful modality for the diagnosis of duplication cyst. On US, the lesion appears as a well-defined anechoic lesion with multilayered wall resembling that of gut (gut signature sign) with associated posterior acoustic enhancement. Gut signature sign is quite specific to this entity, and it can only be visualized on high-resolution US.

The cyst may rarely get infected and lose its characteristic gut signature. In such cases, diagnosis may be difficult and may be confused with other cysts like mesenteric cyst.

CT: CT is not required in most of the cases of gastric duplication cyst, since US can diagnose this entity very well. However, CT can be done in cases where US is equivocal or in complicated cysts. On CT, it appears as a well-defined water density lesion with rim enhancement seen in close relation with the gastric wall. In some cases, the cyst can be confused with pancreatic pseudocyst or gastric stromal tumor.

MRI: It shows the lesion to be hypointense on T1WI and hyperintense on T2WI, with enhancement features similar to those seen on CT. On diffusion-weighted MRI sequences, these lesions do not show restriction except in complicated cysts.

Nuclear scintigraphy: Tc $99 \mathrm{~m}$ labelled pertechnetate scan has a special role in gastric duplication cysts, since these cysts may contain ectopic gastric mucosa, which shows uptake on pertechnetate scan, confirming the diagnosis ${ }^{10,11}$ (-Fig. 4).
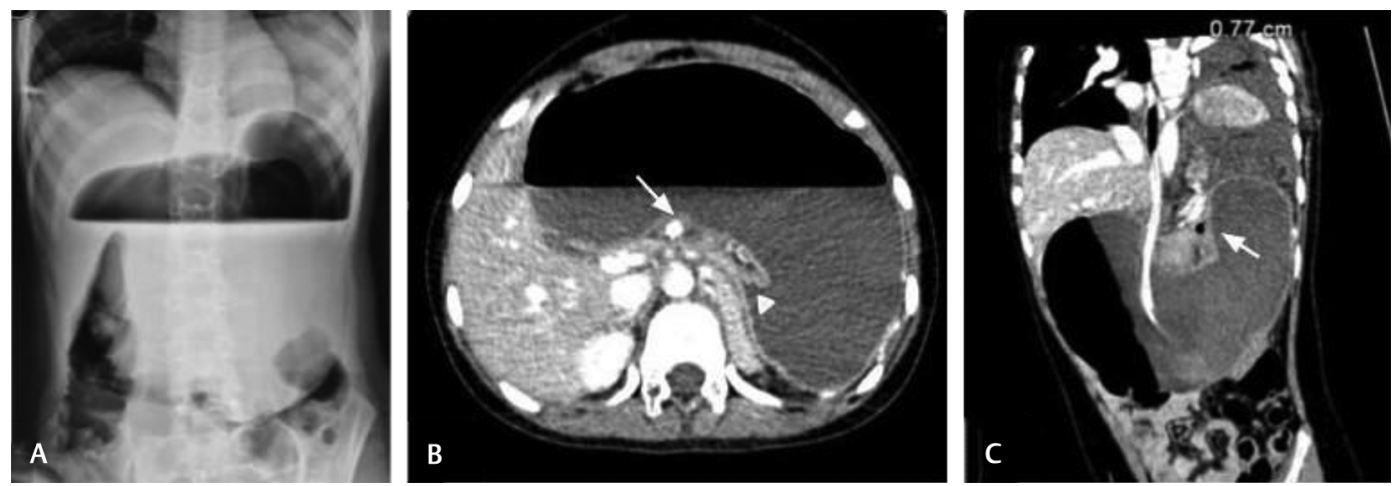

Fig. 3 Gastric volvulus. (A) Abdominal radiograph (AP-erect) shows grossly distended stomach with air fluid level within it. (B) Contrastenhanced computed tomography (CT) axial image showing gastroesophageal (GE) junction (white arrow) and Pylorus (arrow head) at the same level. (C) Coronal CT image showing abnormal position of distended stomach with superiorly displaced pylorus. 


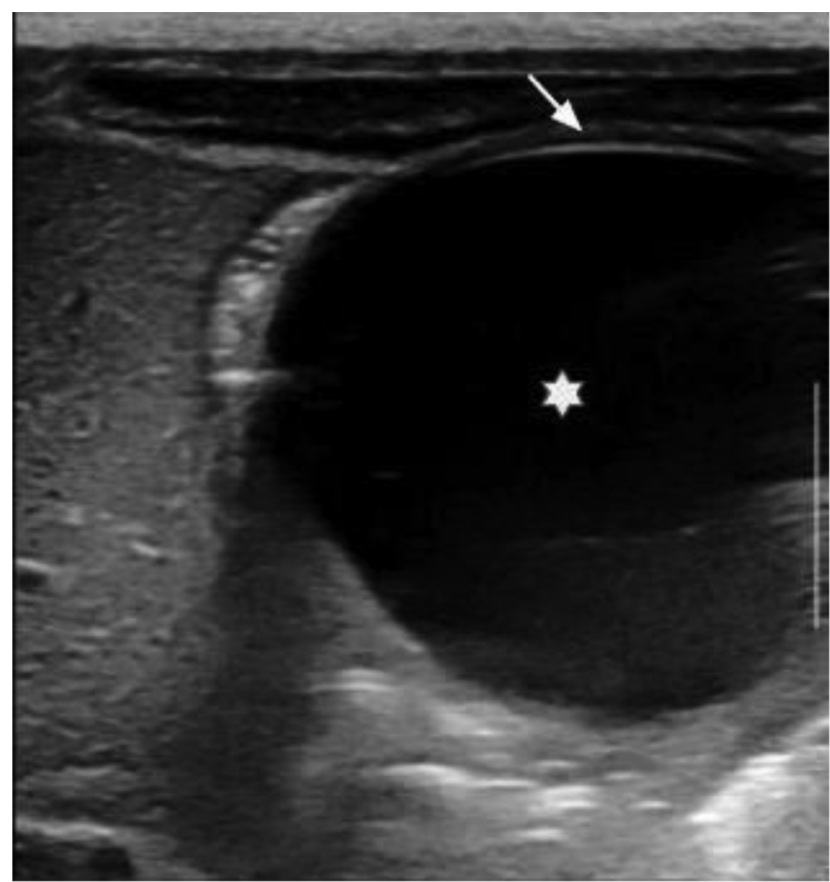

Fig. 4. Gastric duplication cyst. Ultrasound (US) of abdomen shows a well-defined anechoic lesion (star) with posterior acoustic enhancement in the epigastric region, which is compressing and displacing the stomach superiorly. The near wall of the cyst shows the typical gut signature sign (arrow).

\section{Duodenal Atresia/Stenosis/Web Spectrum}

Duodenal atresia is a type of congenital intestinal obstruction, and it is one of the most common causes of fetal and neonatal bowel obstruction. The common location is the second part of the duodenum distal to the ampulla of Vater. Duodenal atresia implies there is complete obstruction of the lumen, whereas duodenal stenosis is the narrowing of the lumen with incomplete obstruction. Duodenal web is a rare abnormality where a membrane causes obstruction of the lumen.

The etiology is the failure of recanalization of the duodenum occurring between the 8th and 10th week of gestation, leading to either incomplete obstruction in the form of stenosis or web, or it may lead to complete obstruction like atresia.

Radiograph: Abdominal radiograph is of great value and shows the classical "double bubble" sign which is diagnostic of this condition. Left-sided and proximal bubble represents the gas in the distended stomach, and the right sided one represents the air in the duodenal bulb with absence of gas shadow distally.

US: Antenatal US performed in the second trimester can help to diagnose this condition in the intrauterine life. US can show two fluid filled loops-stomach and proximal duodenum, respectively. However, this should be interpreted cautiously, as this finding may also be seen in cases of slow peristalsis.

Barium study: Barium meal study can show distended stomach and proximal duodenum with nonpassage of contrast distally. Barium meal may help in differentiating this entity from midgut volvulus, which needs emergency intervention. The findings on barium are dependent on the type of obstruction, namely, stenosis, web and atresia. Duodenal web shows classical "windsock sign" on barium meal study. This finding represents the web in the duodenum, which projects into the duodenal lumen distally, often into the second and third part, because of the stretching of the web by peristalsis.

CT and MRI are usually not necessary and indicated only in cases where there is a diagnostic dilemma ${ }^{12,13}(\sim$ Fig. 5 and $\mathbf{6}$ ).

\section{Annular Pancreas}

Annular pancreas is a congenital morphological malformation involving the pancreas and characterized by pancreas encircling the second part of the duodenum partially or completely. Depending on the involvement, they are divided into two types: complete and incomplete. It is associated with other anomalies such as Down's syndrome, duodenal atresia, and Hirschsprung disease. Annular pancreas results form the failure of rotation of its ventral bud with duodenum leading to encasement of duodenum.

Affected infants present with feeding difficulty and vomiting due to severe duodenal obstruction that requires immediate surgical intervention. The milder variant, namely, incomplete annular pancreas usually does not cause significant symptoms and diagnosis may be delayed till later life.

Radiograph: Abdominal radiograph performed can mimic the findings of duodenal atresia, as it also causes double bubble sign.

US: is of great help in suspected cases of annular pancreas. The pancreatic tissue may be seen encircling the collapsed duodenum in the centre, with proximal dilatation of stomach and duodenum. In incomplete types, the pancreatic parenchyma may show a beak of tissue extending anterior and posterior aspect and showing claw-like appearance.

$C T$ : CT performed in suspected cases can show encasement of the collapsed second part of duodenum by the pancreatic tissue completely or partly around it. Contrast CT shows homogeneously enhancing pancreas around the duodenum.

MRI: The imaging findings are similar to $\mathrm{CT}$; however, the advantage of MRI is in assessing the pancreatic ductal anatomy. Pancreatic duct may be seen on magnetic resonance cholangiopancreatography (MRCP), encircling the duodenum $^{14}$ (-Fig. 7).

\section{Malrotation/Ladd's Band}

Malrotation is a congenital abnormality involving the abnormal position of duodenojejunal (DJ) junction, which may lead to midgut volvulus. Duodenum normally rotates 270 degrees in anticlockwise direction during the first 12 weeks of embryogenesis to reach its normal expected position, whereas in malrotation, there is incomplete rotation, leading to abnormal position of the small bowel loops ( $\mathbf{- F i g . ~ 8 ) . ~}$

Malrotation can be associated with Ladd's band, which extends from abnormally positioned cecum to liver crossing the duodenum and may result in obstruction. 

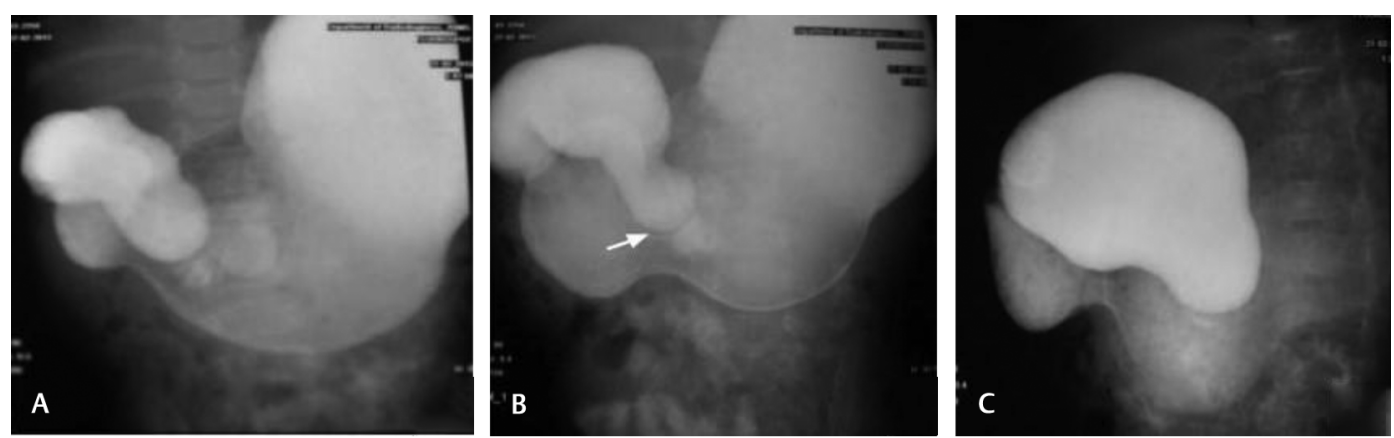

Fig. 5 Duodenal web. (A-C) Frontal and oblique view of barium meal study show caudal ballooning of diaphragm (white arrow), which is seen as a thin filling defect, projecting into the distal duodenum ("windsock sign").
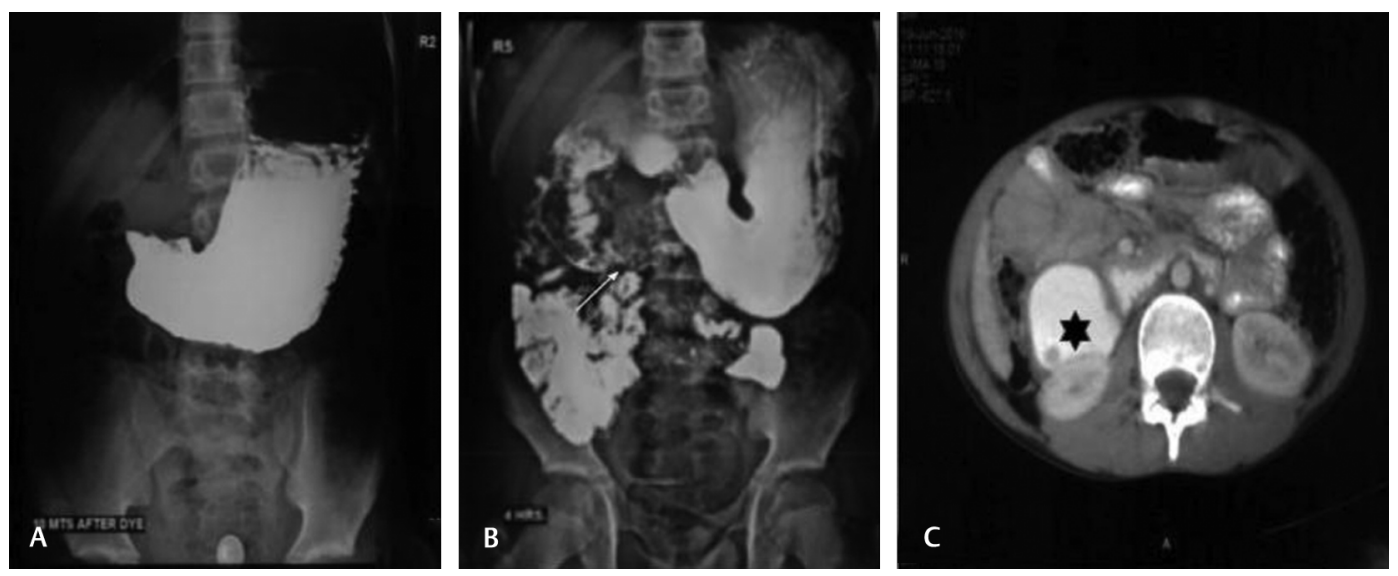

Fig. 6 Duodenal stenosis. (A) Barium meal at 10 minutes after barium ingestion shows normally distended stomach. (B) Delayed phase after 4 hours shows persistent narrowing at D2-D3 junction (white arrow) with proximal dilatation. (C) Contrast-enhanced CT abdomen (axial plane) shows distended D2 part (asterisk) of duodenum with narrowing at D2-D3 junction.
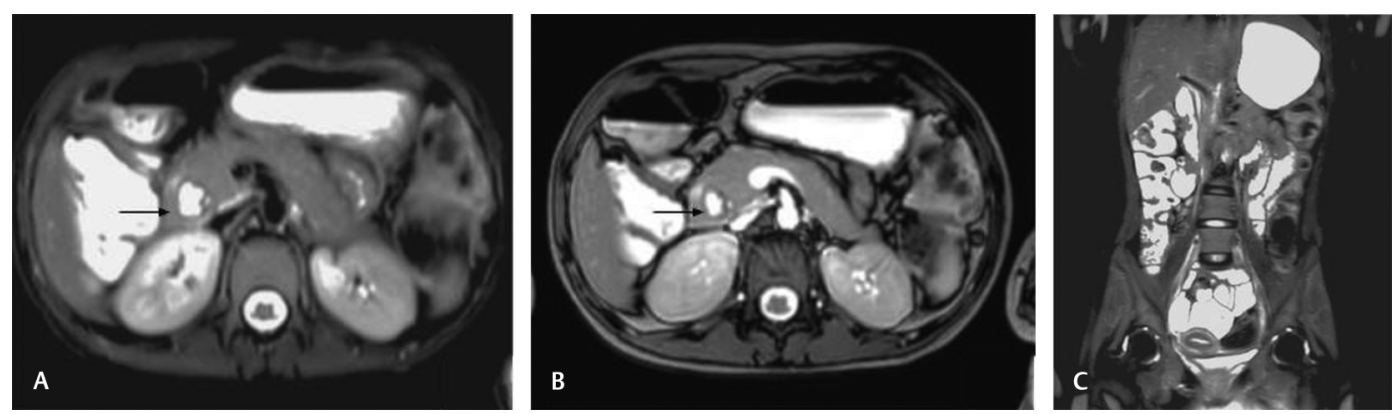

Fig. 7 Annular pancreas. (A, B) Axial T2 fat suppressed (FS) and balanced turbo field echo (BTFE) images, respectively, showing pancreatic parenchyma (arrow) encasing the duodenum completely. (C) Coronal T2FS image showing pancreatic tissue causing narrowing at D2 part of duodenum with proximal dilatation.

Clinical features may vary according to the age, ranging from acute abdominal pain and vomiting to mild intermittent pain and malabsorption.

Radiograph: Abdominal radiograph may be apparently normal, or sometimes when associated with volvulus, there may be features of obstruction like dilated stomach and proximal duodenum.

Barium study: Barium meal follows through is the gold standard in the diagnosis of malrotation and has high sensitivity in identifying it. In frontal projection, the normal DJ junction is located to the left of the left pedicle of the vertebra, whereas in malrotation, the DJ junction lies in midline or to the right of the pedicle. In lateral projection, the normal position of DJ junction is posterior and superior to the duodenal cap. Similarly, cecum should be fixed in the right iliac fossa; however, in malrotation, cecum may be placed more medially. This normal positioning of the DJ and cecum is responsible for the optimal length of mesenteric root. In patients with malrotation because of abnormal position of DJ and cecum, the mesenteric root narrows, predisposing it to volvulus. When associated with volvulus "corkscrew" appearance of the duodenum, proximal dilated bowel loops can be seen. 
US: performed in the transverse plane in a normal child shows superior mesenteric vein (SMV) located to the right side of superior mesenteric artery (SMA) and the third part of duodenum crosses them posteriorly. In malrotation, SMV lies either directly anterior or to the left of the SMA. When midgut volvulus is present, US shows a typical "whirlpool sign" seen as clockwise rotation of SMV, small bowel and mesentery around the centrally placed SMA. SMV may be compressed in some cases, and in such cases, only the mesentery and small bowel rotation around SMA are seen.

CT/MRI: The cross-sectional modalities are not routinely performed, but when there is suspicion of complication, these modalities are indicated. Malrotation may also be incidentally detected in CT/MRI done for another cause ${ }^{15}$ ( Fig. 9).

\section{Preduodenal Portal Vein}

It is a rare anomaly where the portal vein courses anterior to the duodenum instead of its normal posterior location. The affected children are mostly asymptomatic and are incidentally detected on imaging for some other causes.
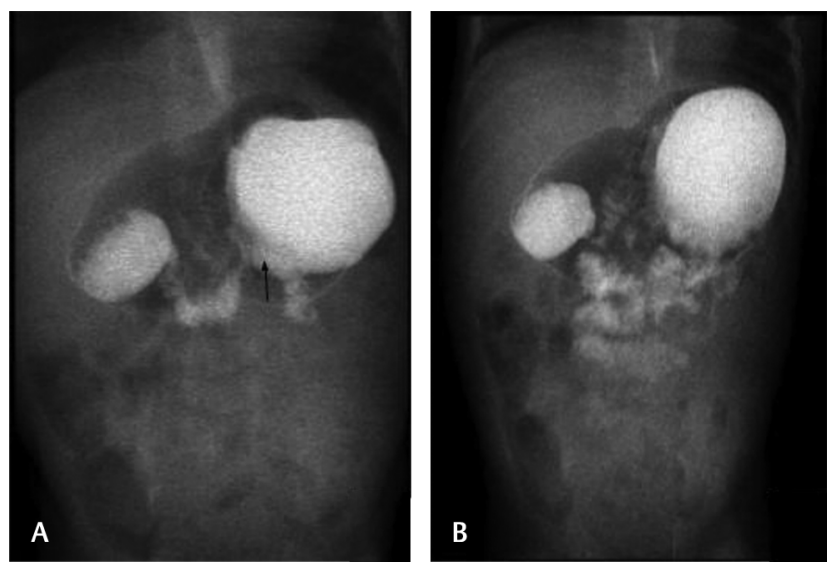

Fig. 8 Normal duodeno-jejunal (DJ) position on barium meal follow through (BMFT). (A) One early fluoroscopic image obtained during BMFT study shows normal position of DJ junction to the left of left pedicle of vertebra. (B) Follow-up image shows small bowel loops located centrally and to the left of midline.
However, rarely this entity can produce features of duodenal obstruction.

It is typically associated with other congenital anomalies such as heterotaxia or polysplenia syndrome, situs inversus, cardiac defects, malrotation, biliary or duodenal atresia and annular pancreas.

During embryological development, the two parallel vitelline veins are joined by three interconnecting veins-the cephalad branch within the liver, the middle branch posterior to duodenum, and the caudal branch anterior to duodenum. Cephalad and caudal branches disappear eventually, leaving the middle branch, which forms the normal portal vein. In preduodenal portal vein (PDPV), there is disappearance of the cephalad and middle branch, leaving the caudal branch to form the PDPV.

Radiograph: The abdominal radiograph may be normal or show dilated stomach and proximal duodenum in cases where there is associated obstruction.

US: shows normal pylorus and a preduodenal portal vein as an anechoic structure on B mode with color flow on color Doppler study.

CT/MRI: In cross-sectional studies like CT/MRI, sometimes it is incidentally detected in asymptomatic patients. The imaging features are similar to those on US, showing anterior position of portal vein in relation to the duodenum ${ }^{16}(-$ Fig. 10).
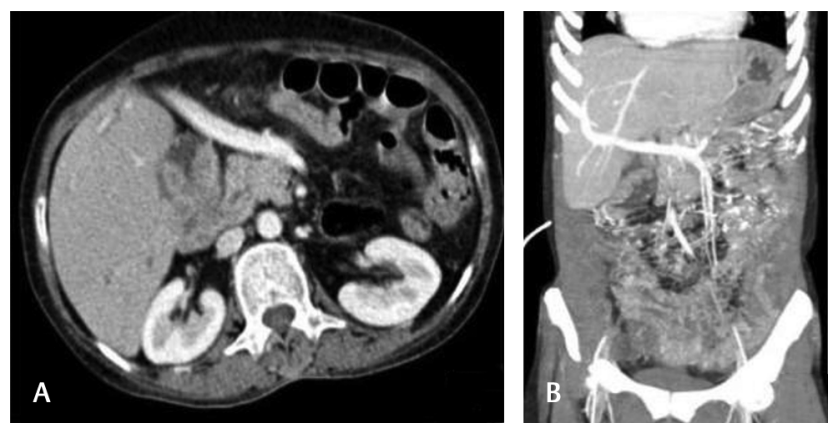

Fig. 10 Preduodenal portal vein. (A) Axial contrast-enhanced (CE) abdomen showing portal vein coursing anterior to the duodenal loops. B. Coronal maximum intensity projection (MIP) image showing the portal vein entering the right lobe of liver instead of porta.
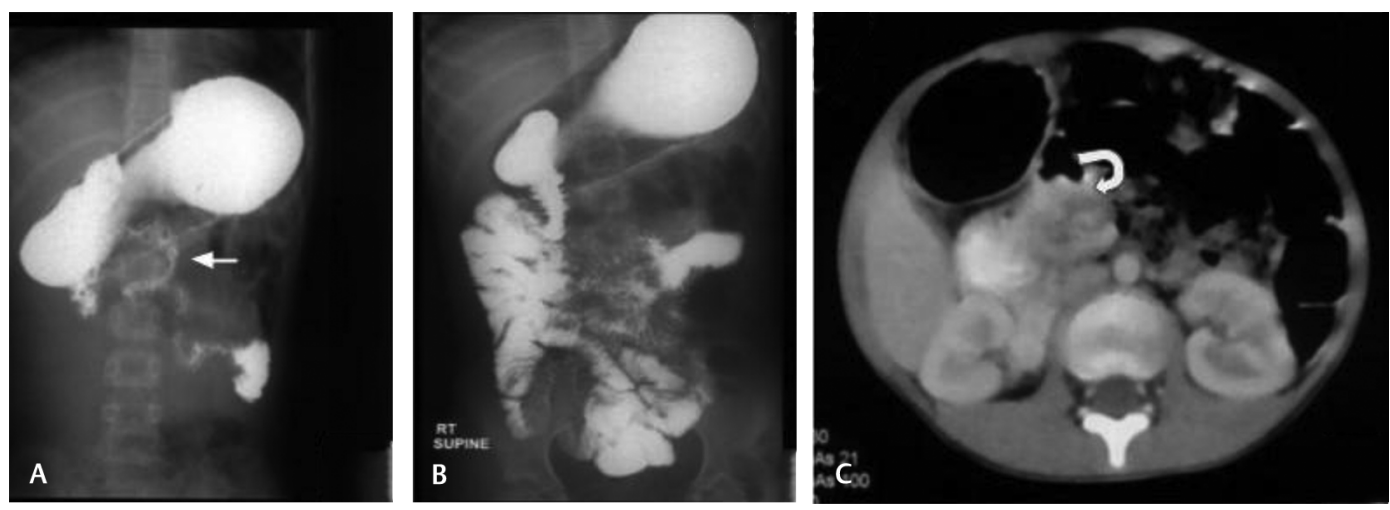

Fig. 9 Malrotation associated with midgut volvulus. (A, B) Barium meal follow through (BMFT) showing abnormal location of duodeno-jejunal (DJ) junction with corkscrew appearance (white arrow) of the duodenal and proximal jejunal loops. The small bowel loops are seen predominantly in the right side of the abdomen. (C) Contrast-enhanced CT abdomen showing whirlpool sign (curved arrow) with dilated duodenum. 


\section{Inflammatory Conditions}

\section{Eosinophilic Gastroenteritis}

It is an unusual benign inflammatory disorder, which is characterized by infiltration of the esophagus, stomach as well as small bowel by eosinophils. Affected children have a strong history of asthma and atopy. They usually have elevated levels of serum IgE and peripheral eosinophilia and show good response to steroids.

According to Klein classification, eosinophilic Gastroenteritis (EG) is classified into three types, namely, mucosal (most common), muscular and serosal. Gastric antrum is the most commonly involved part, showing characteristic nodularity and thickening of mucosal lining. Clinical features include nonspecific findings like nausea, vomiting, and abdominal pain.

Imaging features are not very characteristic; final diagnosis needs confirmation by endoscopic biopsy. Biopsy is usually negative in serosal type.

Radiograph: Abdominal radiograph is not useful and may be apparently normal in these children.

Barium study: Double contrast barium study may show thickening and lacy appearance of gastric antral mucosa; however, this is not specific for eosinophilic gastritis and can be seen in any type of gastritis.

CT/MRI: The findings of EG vary according to the type, as mucosal form may show fold thickening and polyps. Muscular type may show wall thickening and stricture formation, leading to proximal obstruction. Halo sign and arachnid limb (spider leg) like sign are also described, which can be used to differentiate it from lymphoma. Serosal type may show ascites, omental thickening, serosal enhancement, and necrotic mesenteric lymphadenopathy ${ }^{17}$ (- Fig. 11).

\section{Crohn's Disease}

Crohn's Disease (CD) is a type of inflammatory bowel disease, with involvement anywhere from mouth to anus. The involvement of the upper GI tract is less common in comparison to small bowel involvement where distal ileum is the most common site involved.

The upper GI involvement is more common in the pediatric age group compared to adults, with the stomach being the most common site, followed by esophagus and duodenum.
Focally enhanced gastritis is a form of gastric inflammation seen in pediatric inflammatory bowel disease (IBD), showing focal inflammation of gastric mucosa in addition to the presence of granulomas in histology. Duodenal involvement can be of three types: contiguous antroduodenal disease (common), isolated involvement of descending part of duodenum, and distal duodenal type.

Radiograph: Abdominal radiograph is not of much use and usually will not reveal any abnormality except in severe disease where classic ram`s horn appearance (funnel-shaped deformity of diseased antrum and duodenal bulb) can be seen

CT: Enterography is the optimal technique to evaluate the bowel loops in distended state. CT enterography (CTE) may show features of focal gastritis: mucosal nodularity which shows enhancement. In severe cases, concentric thickening of the pyloric part, with associated gastric outlet obstruction, is seen.

MRI: MR enterography (MRE) is usually preferred over CT in children both at baseline and follow-up. It is better than CT for the evaluation of disease activity and response to therapy, as it provides multiple paradigms for assessing the disease. ${ }^{18}$

\section{Chronic Granulomatous Disease}

Chronic granulomatous disease (CGD) is an immunodeficiency disorder characterized by phagocytic dysfunction where phagocytes fail to kill catalase positive organisms such as staphylococcus and aspergillus. This leads to recurrent infection, inflammation, and granuloma formation.

It is an inherited disorder most commonly transmitted by $\mathrm{X}$-linked recessive inheritance; however, an autosomal variant has also been described.

Phagocytic nicotinamide adenine dinucleotide phosphate (NADPH) when activated releases free radicals in the phagosome, thus helping in killing the microbes. In CGD, NADPH mutation results in failure of free radical formation, thus leading to phagocytic dysfunction. Nitroblue tetrazolium and dihydrorhodamine reductase flow cytometry studies are used for the diagnosis of CGD.

CGD shows multisystem involvement including chest, lymph node, GI tract, liver, spleen, bones, urinary tract, and central nervous system (CNS).

In the GI system, it can involve any part.
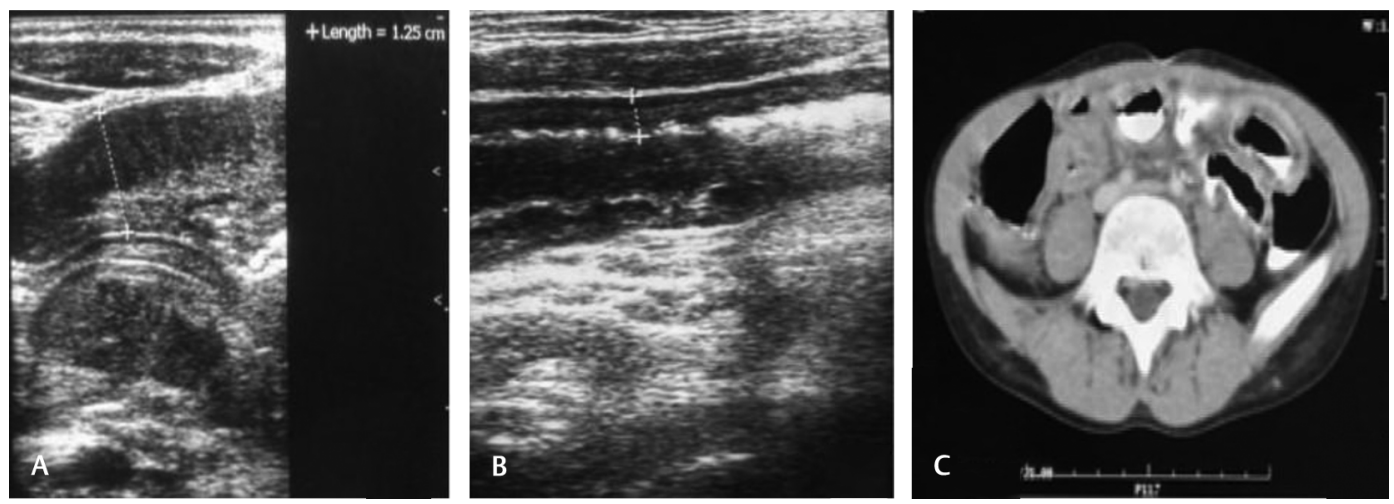

Fig. 11 Eosinophilic gastroenteritis. (A, B) Ultrasound image shows circumferential mural thickening of the stomach wall and small bowel loops. (C) Contrast-enhanced CT showing diffuse circumferential wall thickening of multiple small bowel loops. 
Stomach when involved may give rise to gastric outlet obstruction, due to concentric thickening of pylorus.

Radiograph: Abdominal radiograph may show distended stomach which can mimic other conditions causing gastric outlet obstruction.

US: may show concentric wall thickening of the antropyloric region with proximal distended stomach. However, this finding is quite nonspecific and can be seen in other conditions such as eosinophilic gastritis, peptic ulcer disease, and CD.

CT/MRI: The cross-sectional imaging modalities are particularly useful when multisystem involvement is present. It may show wall thickening of the antropyloric region of the stomach, which is a nonspecific feature. ${ }^{19}$

\section{Tuberculosis}

Tuberculosis (TB) of the GI tract commonly involves the ileocecal junction. Gastroduodenal tuberculosis is rare even in endemic areas. Gastroduodenal TB can occur as a direct extension from the adjacent tubercular disease or due to hematogenous spread. Gastric antrum along the greater curvature is the most common location to be involved, which may extend to proximal duodenum. It usually starts as a submucosal disease, leading to mucosal ulceration resembling carcinoma and eventually healing with fibrosis. Fibrotic TB can resemble linitis plastica, or lymphoma.

Radiograph: It may be normal in early disease. In advanced cases, distended stomach can be seen, suggesting gastric outlet obstruction.

Upper GI study: The antral part may show features of ulceration which may mimic adenocarcinoma. Narrowing and gastric outlet obstruction due to fibrosis are other features which may be seen.

US: may show wall thickening at the involved antral part and the proximal duodenum. Enlarged necrotic regional lymph nodes may also be seen.

CT/MRI: Cross-sectional studies are better in showing the circumferential wall thickening of the antrum, stomach distension, and associated necrotic nodes, which may show peripheral rim enhancement ${ }^{20-22}$ ( - Fig. 12).

\section{Neoplastic Conditions}

Lymphoma:

Primary lymphoma of the GI tract is rare, and it is usually involved secondarily in cases with multisystemic involvement. Most common type of GI lymphoma is nonHodgkin's lymphoma (NHL). Primary GI lymphoma most commonly involves the stomach and is common in the pediatric age group. Risk factors for gastric lymphoma include $\mathrm{H}$. pylori infection, immunosuppression after solid organ transplantation, celiac disease, IBD, and HIV infection.

Dawson's criteria must be met to diagnose primary gastric lymphoma which include

1. No palpable superficial lymphadenopathy.

2. Whole blood count must be normal.

3. Normal chest radiograph.

4. Predominant involvement of the GI tract.

5. No liver and spleen involvement.

Secondary involvement of the stomach by lymphoma is more common than primary involvement. Most common type of primary lymphoma affecting the GI tract is NHL, of which Burkitt's lymphoma is the commonest. Stomach can be involved by primary Burkitt's lymphoma; however, it is not the most common site.

Clinical presentation includes dyspepsia and epigastric pain. Lymphomas usually do not cause luminal obstruction and hence they rarely present with gastric outlet obstruction.

Barium meal: Double contrast barium studies may show various patterns like polypoidal, ulcerative, and infiltrative patterns, which may be seen with gastric carcinoma as well; however, they are much rarer. Multiple polypoidal masses with central cavitation showing bull's eye appearance may also be seen in gastric lymphoma. Extensive gastric fold thickening with normal preservation of distensibility of stomach is also a feature of gastric lymphoma.
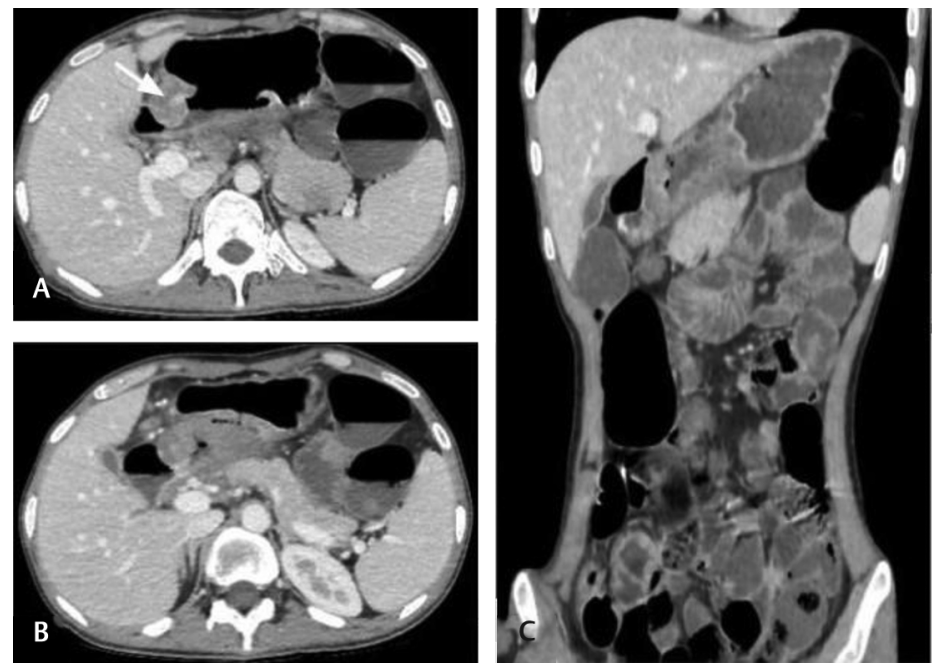

Fig. 12 Gastrointestinal (GI) tuberculosis (TB). (A, B) Axial CT section showing asymmetric wall thickening (straight arrow) in the pyloric region of stomach without obstruction. (C) Coronal image showing asymmetric wall thickening in the pylorus of stomach. (D) Contrast-enhanced CT images at lower abdomen showing short segment small bowel stricture (curved arrow). 
US: Diffuse wall and gastric fold thickening with associated lymph nodal enlargement can be seen on US.

CT: Diffuse gastric wall and mucosal fold thickening with preservation of the fat plane and adjacent structure and absence of luminal obstruction is typical of the gastric lymphoma. Gastric adenocarcinoma can also present with gastric wall thickening; however, the fat plane with the adjacent structure is usually lost and usually presents with obstruction. The lymph nodal involvement in lymphoma is typically discrete, homogeneous, noncalcified and usually nonnecrotic.

MRI: MRI is not routinely performed for the evaluation of gastric lymphoma; however, it can be used as an alternative modality to CT in follow-up cases $^{23}$ ( - Fig. 13).

\section{Gastrointestinal Stromal Tumor}

Gastrointestinal stromal tumor (GIST) is a mesenchymal tumor arising from interstitial cells of Cajal and is rare in the pediatric age group. Pediatric GIST may arise sporadically or can be associated with syndromes like Carney's triad (GIST, pulmonary chondroma and paraganglioma), CarneyStratakis syndrome (GIST with paraganglioma) and neurofibromatosis type 1.

GIST occurring in children is quite distinct from its adult counterpart in immunohistochemistry, natural history, and treatment response. Genetically, 85\% of pediatric GIST show mutation in succinate dehydrogenase (SDH) complex and lacks mutation in KIT/PDGFRA (seen in adult type). Tumors lacking the KIT/PDGFRA mutation are also known as "wild-type" (WT) GIST.

CT: remains the important modality in the diagnosis of the GIST. Large $(>5 \mathrm{~cm})$ lesions are typically exophytic and hypervascular with heterogeneous enhancement on contrast-enhanced $\mathrm{CT}$ along with necrosis and cavitation, whereas small lesions $(<5 \mathrm{~cm})$ are typically submucosal or endoluminal polypoid masses that show homogeneous contrast enhancement. Metastatic lesions usually show arterial enhancement with washout in portal and venous phase. The SDH deficient type of GIST seen in the pediatric age group is seen more often in girls and can be associated with lymph node metastases. Resectable lesions are treated surgically, whereas advanced lesions are treated with imatinib with or without surgery. When imatinib is used, Choi's criteria are used for response assessment rather than the conventional RECIST criteria.
MRI: is used as an alternative modality to $\mathrm{CT}$ to reduce the risks of radiation. Additionally, MRI with diffusion-weighted imaging has been shown to aid in the adult population in prediction of high-risk tumors. Low apparent diffusion coefficient (ADC) values and intratumoral cysts are found to be associated with high-risk tumors. However, this has not been studied in the pediatric population.

Contrast-enhanced US (CEUS): enables the evaluation of hypervascular as well as hypovascular small tumors. The typical characteristics of GIST on CEUS are hyperenhancement on arterial phase and hypoenhancement on portal venous phase. CEUS has also been shown efficacious to assess disease progression and treatment response when tyrosine kinase inhibitors (TKIs) are used for treatment,

Positron emission tomography-CT (PET-CT):not only has high sensitivity in detecting metastasis (80-90\%), but also helps in response assessment of GISTs treated with TKIs ${ }^{24}$ (-Fig. 14).

\section{Adenocarcinoma}

Gastric adenocarcinoma is rare in the pediatric age group. Etiology of the pediatric adenocarcinoma is unclear; however, the current understanding is that it may be associated with genetic mutation.

The most important risk factor for pediatric adenocarcinoma is Helicobacter pylori infection, which can cause chronic active inflammation in the gastric mucosa, commonly in the antrum.

The most common presentations in pediatric gastric adenocarcinoma are abdominal pain and vomiting. Other symptoms include hematemesis, melena, and weight loss. Mucinous adenocarcinomas are seen more often in the younger age group.

Barium meal: As discussed previously, similar to lymphoma, adenocarcinoma of the stomach may show ulcerative, polypoidal, and infiltrative patterns on double contrast barium meal study.

CT: Contrast-enhanced CT may show diffuse or asymmetric focal gastric wall thickening, most commonly in the antral part. There may be associated loss of fat plane with the adjacent structures and gastric outlet obstruction when pylorus is involved. Mucinous tumors frequently show calcification. There may be associated lymphadenopathy, omental deposits, and ascites in advanced cases.
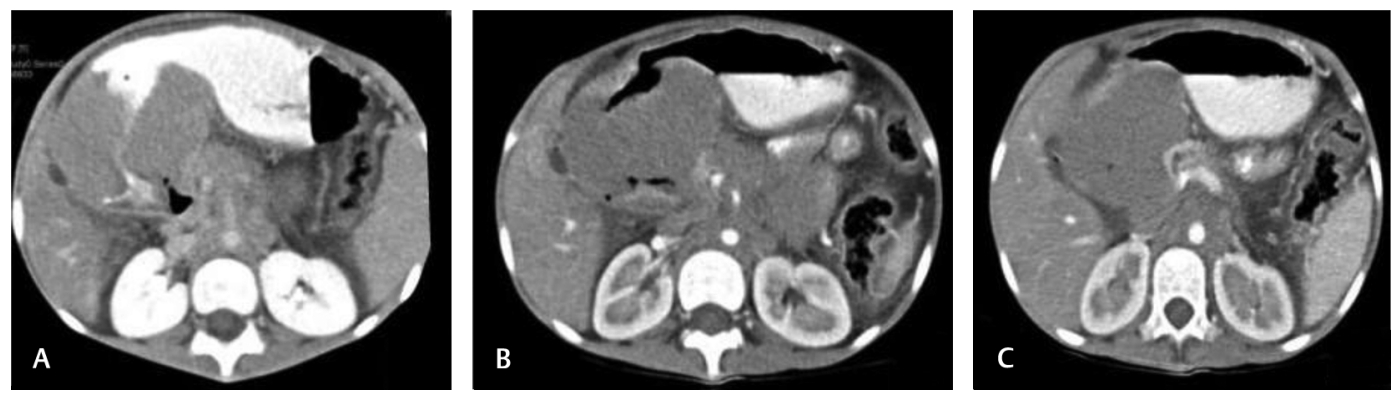

Fig. 13 Gastric lymphoma. (A-C) Axial computed tomography images showing gross thickening of the pyloric region of the stomach without luminal obstruction. There are enlarged retroperitoneal lymph nodes. 

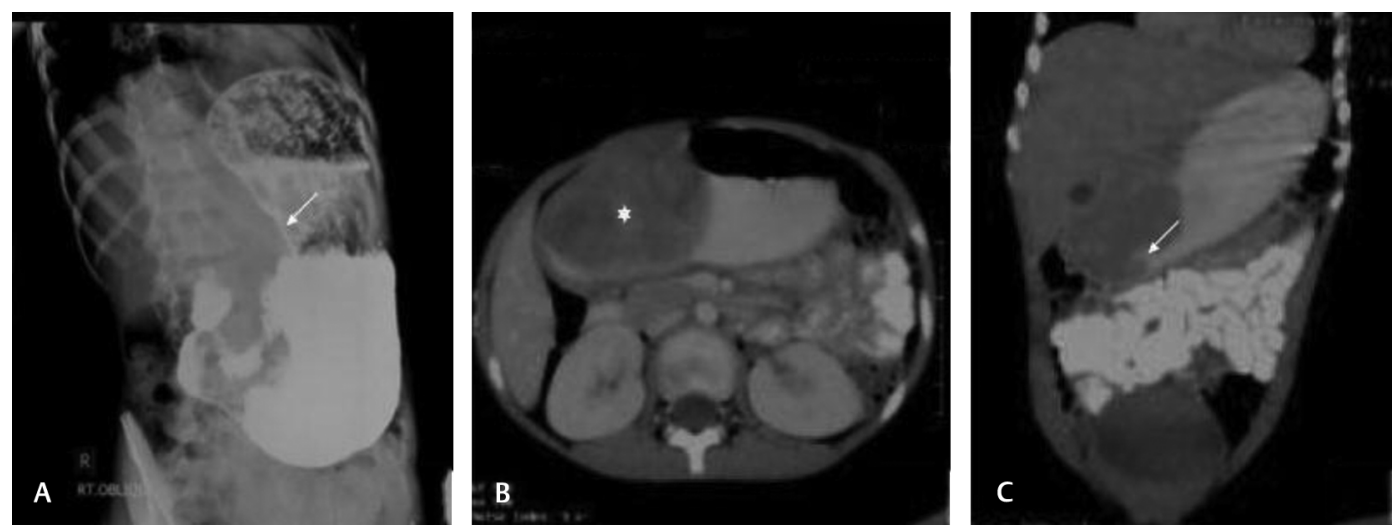

Fig. 14 Gastrointestinal (GI) stromal tumor. (A) Barium meal study showing filling defect projecting endophytically from the gastric wall along the lesser curvature (arrow). (B) Contrast-enhanced computed tomography (CT) abdomen axial image showing intraluminal heterogeneously enhancing soft-tissue (asterisk) mass in the stomach. (C) Coronal contrast-enhanced CT image showing soft-tissue mass arising from the lesser curvature of the stomach showing ulceration (arrow).
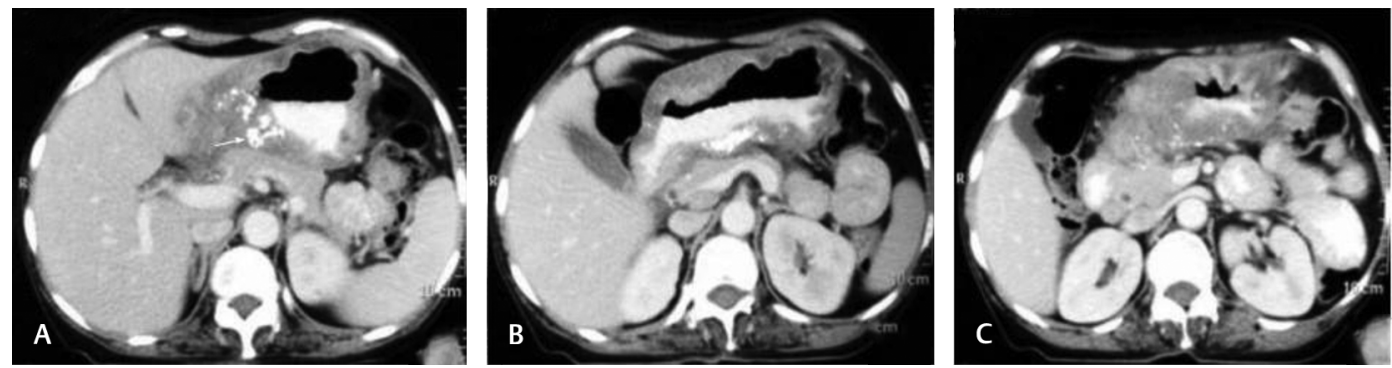

Fig. 15 Gastric adenocarcinoma. (A-C) Axial computed tomography images show asymmetric circumferential wall thickening involving body and antrum of the stomach with areas of coarse calcifications (arrow) within, consistent with mucinous variety of adenocarcinoma.

MRI: can be used as an alternative modality to avoid radiation and can also be used as a problem-solving modality whenever there is some diagnostic dilemma on $\mathrm{CT}^{25}$ (-Fig. 15).

\section{Teratoma}

Teratomas are benign neoplasms arising from pluripotent cells, commonly seen in the pediatric age group. It may be gonadal or extragonadal. Extragonadal teratomas are more frequently seen in newborns, infants, and toddlers, whereas gonadal teratomas occur more often in older children.

Among GI teratomas, the stomach is the most commonly involved site. It commonly arises from greater curvature but can also arise from other parts as well.

These tumors usually contain derivatives of all three germ cell layers; however, the presence of two layers are enough for making the diagnosis histologically.

Presenting complaints are a palpable mass in the upper quadrant, feeding difficulty, vomiting and respiratory distress due to the mass effect over the diaphragm.

Radiograph: Abdominal radiograph may show soft-tissue mass in the epigastric region with areas of coarse calcifications, which are seen in 35 to $60 \%$ of the cases. Rarely, teeth and osseous structures may be seen within the soft-tissue mass. Occasionally, teratomas with large amounts of fat can present as lucent masses.
US: On US, the appearance may range from cystic, solid-cystic to completely solid masses. Cystic lesions are seen as anechoic lesions with well-defined margins. Foci of calcification are seen as hyperechoic areas with posterior acoustic shadowing on US. Solid masses are usually seen as heterogeneous masses.

CT: is particularly valuable in the diagnosis of gastric teratoma. It is seen as well-defined soft-tissue mass noted in the upper abdomen compressing and abutting the greater curvature of the stomach, with fat density areas and foci of coarse calcifications within ${ }^{26}$ (-Fig. 16).

\section{Hemangioma}

Hemangiomas are benign vascular tumors that can occur anywhere in the GI tract. Small intestine is the most common location. Gastric hemangiomas comprise $1.6 \%$ of all benign gastric tumors.

Presence of multiple hemangiomas is usually associated with syndromes like Klippel-Trenaunay syndrome, Maffucci syndrome, blue rubber-bleb nevus syndrome, and OslerRendu-Weber syndrome. These tumors can occur at any age but are most commonly seen in the pediatric age group, and they sometimes involute with time.

Clinical presentation includes GI bleed, intussusception, and abdominal pain. Upper GI endoscopy may show reddish lobulated submucosal mass in the stomach. Endoscopy also has a therapeutic role in resection of gastric hemangioma. 

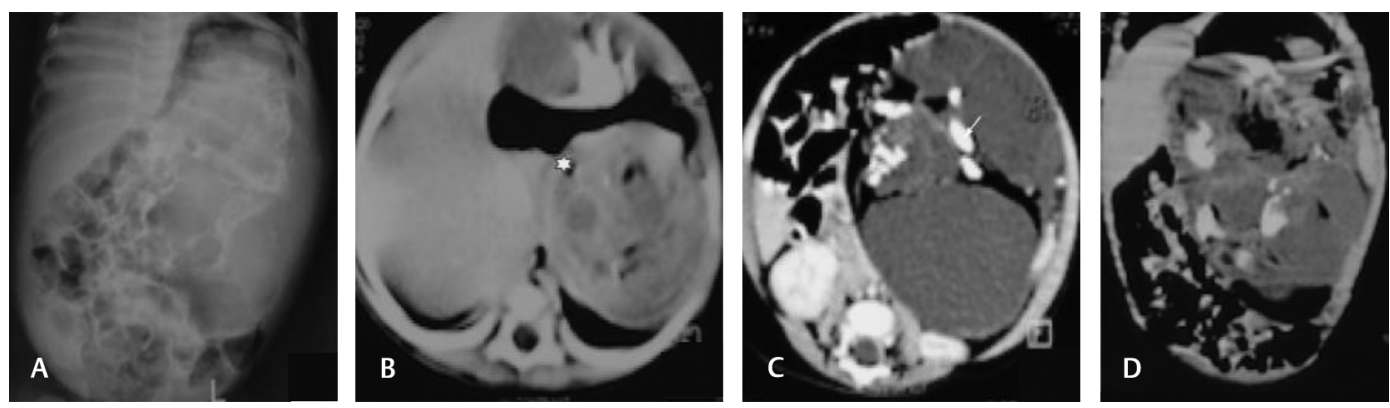

Fig. 16 Gastric teratoma. (A) Abdominal radiograph showing large soft-tissue mass in the left half of abdomen with areas of calcifications within. (B, C) Axial computed tomography (CT) image showing heterogeneously enhancing soft-tissue mass with areas of calcifications (arrow) and fatty attenuation seen on either side of the compressed stomach (asterisk). (D) Coronal contrast-enhanced CT image showing the large solid cystic mass seen occupying the left half of abdomen with displacement of bowel loops inferiorly.
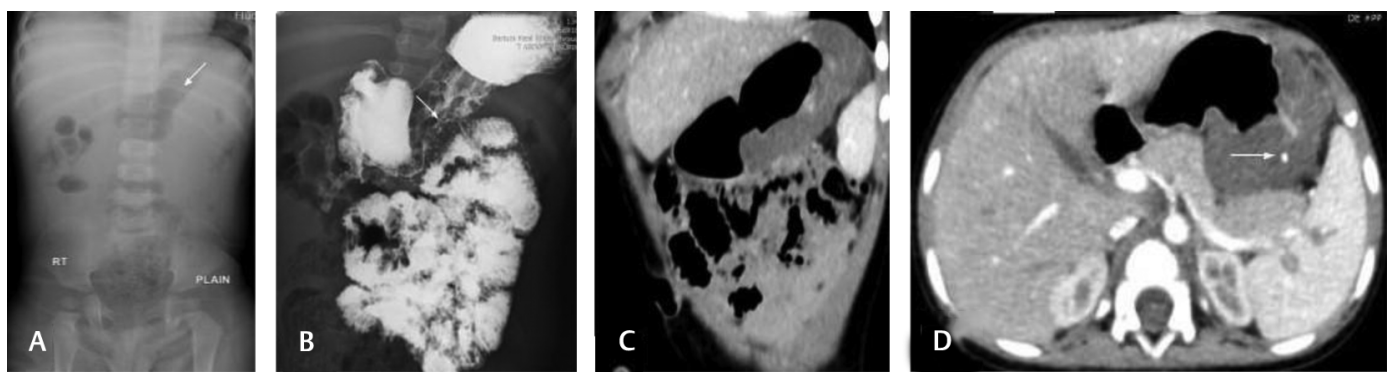

Fig. 17 Gastric hemangioma. (A) Abdominal radiograph showing luminal narrowing of the stomach (arrow). (B) Barium meal follow through showing intraluminal filling defect with luminal compromise of the stomach. (C, D) Coronal and axial computed tomography images showing focal thickening of posterior wall of body and fundus of stomach with a phlebolith (arrow).

Barium meal: The findings are quite nonspecific which may show intraluminal filling defect suggestive of mass.

CT: Contrast-enhanced CT can show avidly enhancing softtissue mass in the lumen of the stomach suggestive of vascular tumor; in addition, it may show phleboliths which are diagnostic of this condition ${ }^{27}$ ( - Fig. 17).

\section{Polyp}

Juvenile polyposis syndrome is one of the most common polyposis syndromes, which can present with multiple polyps in the small bowel. However, involvement of the stomach and duodenum is rare, and if at all seen, they are usually seen in the antrum of the stomach.

Familial adenomatous polyposis can also show gastric polyps which are most commonly seen in the fundus and body of the stomach.

Large polyps sometimes can prolapse and cause pyloric obstruction. Inflammatory fibroid polyps (IFPs) occur anywhere in the GI tract, and the majority of them occur in the gastric antrum (75\%), followed by the gastric body and fundus. These are submucosal lesions which can mimic adenomatous polyps and other intraluminal stromal tumors.

Double-contrast barium meal: It may show multiple intraluminal polyps as soft-tissue filling defects projecting into the lumen. Multiple signs described for polyps include bowler's hat sign and target sign which can aid in diagnosing these polyps.

CT: On contrast-enhanced CT scan, polyps may be seen as solitary or multiple low attenuation frond-like projections filling the stomach and the duodenum ${ }^{28,29}(-$ Fig. 18).

\section{Miscellaneous}

Bezoar

Bezoars result from accumulation of ingested foreign body material in the GI tract. They are classified based on the content ingested into phytobezoar (fruit or vegetable fiber), trichobezoar (hair), lactobezoar (undigested milk), and pharmacobezoar (unabsorbed medications). Trichobezoars are seen most commonly in young girls who have the habit of ingesting hair or in patients with psychiatric illness. Hair usually accumulates in the stomach and does not pass into the small intestine. Phytobezoar is seen in children with poor chewing ability and ingestion of fiber-rich diet and commonly have previous history of GI surgery. They can also pass into the small intestine and cause small bowel obstruction.

Radiograph: Trichobezoar in the stomach is seen as distended stomach with intraluminal contents and mottled air lucencies suggestive of air trapped within the accumulated foreign body.

Barium meal: Barium studies are used to confirm the bezoar as well as help in differentiation between obstructions caused by a bezoar from those caused by adhesions.

Ultrasound: Bezoar on US are seen as heterogeneous and hyperechogenic mass with marked acoustic shadowing, which is different from the one produced by food particles.

CT: is helpful in the evaluation of small bowel bezoar and may show a well-defined oval intraluminal mass with air bubbles retained within the interstices. CT can also show floating masses at the water-air surface, as bezoars have a lower density than food. 

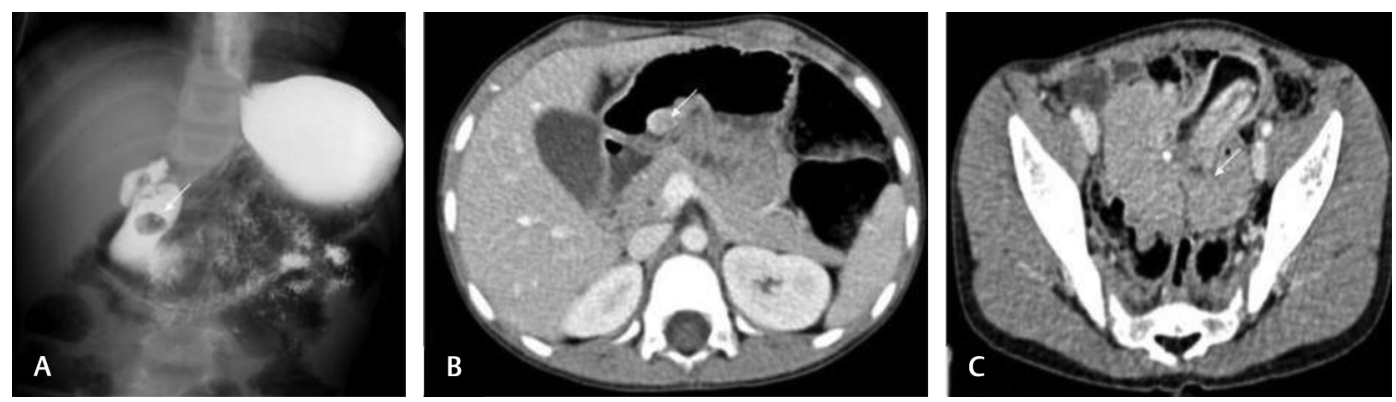

Fig. 18 Peutz-Jegher syndrome. (A) Barium meal image showing oval filling defect (arrow in A) seen in the pylorus of stomach. (B, C) Axial contrast-enhanced computed tomography images showing homogeneously enhancing polyp noted along the posterior wall of stomach (arrow in $\mathbf{B}$ ) and a larger polyp in the sigmoid colon causing intussusception (arrow in C).

MRI: is not useful in the diagnosis because the signal intensity of a trichobezoar is low and easily confused with air. $^{30}$

\section{Duodenal Hematoma/Perforation}

Duodenal injuries in children are commonly due to motor vehicle accidents. Nonaccidental trauma is a common cause in infants and young children. Male children and low socioeconomic status are considered as predisposing factors in these cases.

Radiograph: Abdominal radiograph may show soft-tissues mass between the stomach and the transverse colon.

Pneumoperitoneum/pneumoretroperitoneum may also be seen when there is duodenal perforation, depending on the site of injury.

US: may show duodenal mass with associated free fluid in the abdomen and there may be associated periduodenal hematoma, which is seen as hyperechoic content.

CT: Contrast-enhanced CT may better delineate a welldefined low-to-intermediate density mass seen in the wall of the duodenum. There may be few small foci of air noted in the wall adjacent to mass, and the lesion does not enhance with contrast. Free extraluminal air may be seen if there is duodenal perforation.

MRI: is usually performed for the confirmation of findings when the CT findings are not consistent with hematoma or equivocal. Typical T1 hyperintensity confirms the diagnosis of duodenal hematoma in acute and subacute phases $^{31}$ (-Fig. 19).

\section{Corrosive Stricture}

Ingestion of corrosive substances such as acid or alkali can cause damage to the upper GI tract. Esophagus and/or stomach involvement may occur; hence, it is important to evaluate both of them on imaging. Most commonly responsible acidic agents are the hydrochloric acid or mixture of hydrochloric acid and nitric acid, which upon ingestion cause injury due to coagulative necrosis.

Alkali agents which are frequently used are hydroxides of sodium and potassium. These cause injury due to liquefactive necrosis. Alkali agents cause more damage than the acidic agents.
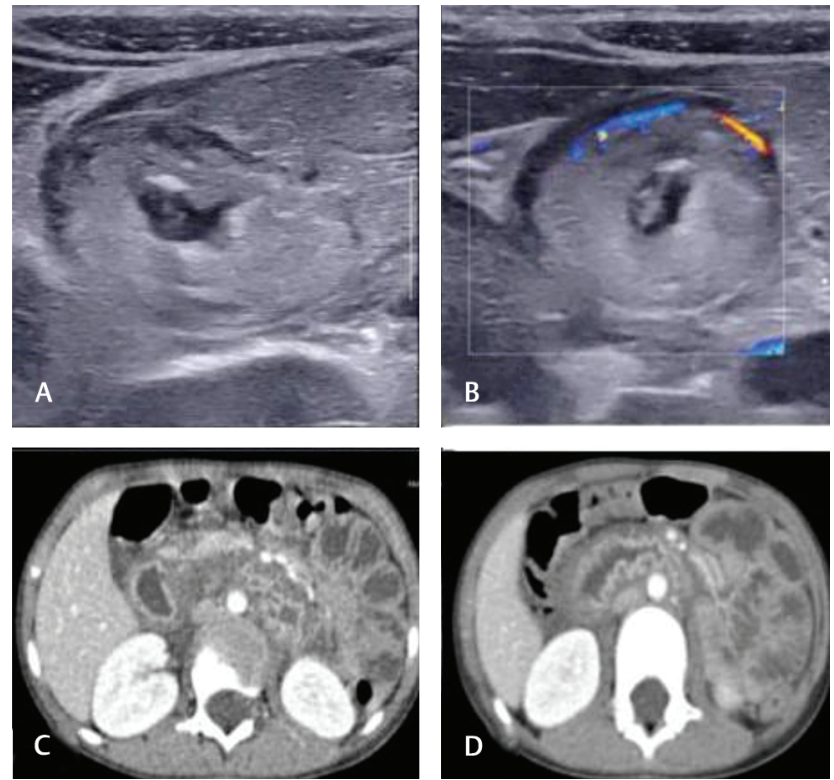

Fig. 19 Duodenal hematoma in Henoch-Schonlein purpura. (A, B) Ultrasound (US) in axial plane showing diffuse circumferential thickening of the duodenal wall, showing no color flow on Doppler in a child with Henoch-Schonlein purpura. (C, D) Contrast-enhanced computed tomography axial images showing circumferential thickening of duodenal wall with maintained stratification.

Radiograph: It is useful in evaluating perforation which can manifest as pneumomediastinum or pneumoperitoneum, depending on the involved site.

Upper GI contrast study: Water-soluble contrast medium is used instead of barium in these patients, so as to avoid the risk of leak and mediastinitis/peritonitis. The imaging findings may vary, depending on the phase of the disease process. In chronic cases, they usually show short- or long-segment smooth concentric narrowing of the esophageal lumen, which is seen most commonly in upper $1 / 3 \mathrm{rd}$ in children. In stomach, antropyloric region is most commonly affected; however, with ingestion of large amounts, there can be involvement of other parts as well sometimes, showing a small contracted stomach with hourglass appearance.

CT/MRI: CT is used in cases with suspected perforation to look for the site and assess the extent of involvement. MRI does not have any additional benefit over CT in these patients $^{32}(-$ Fig. 20). 

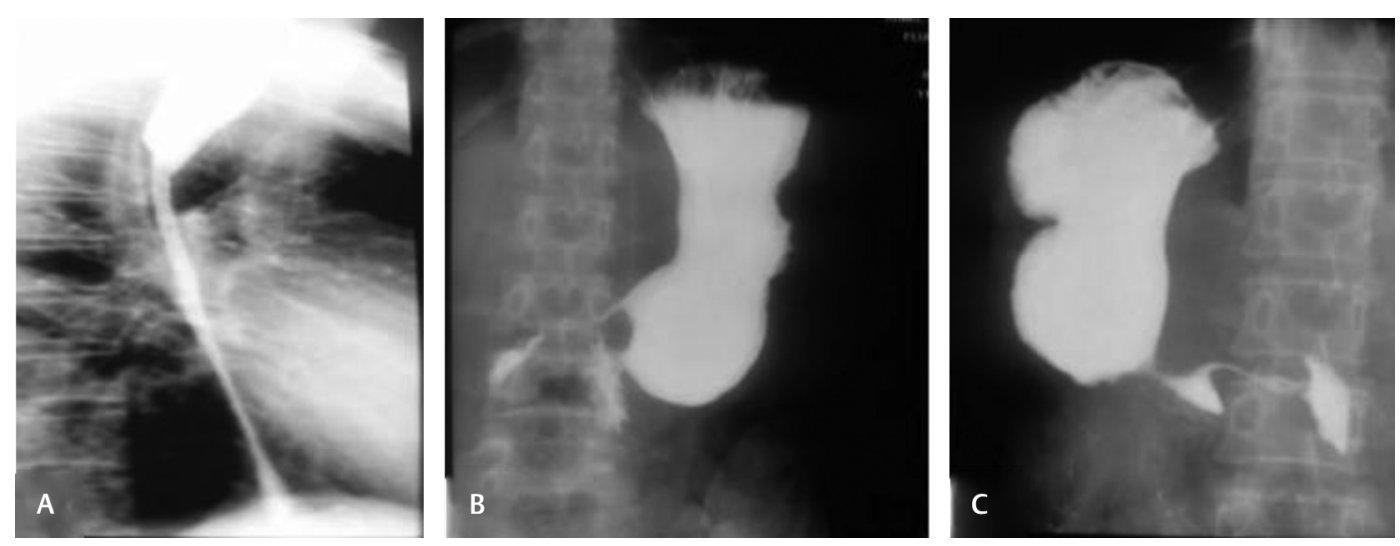

Fig. 20 Corrosive stricture. (A) Fluoroscopic image (lateral view) during contrast swallow of esophagus shows long segment smooth concentric narrowing of the lumen at mid and lower 1/3rd of esophagus with involvement of gastroesophageal (GE) junction s/o benign stricture due to corrosive injury. (B, C) Upper gastrointestinal contrast study shows long-segment concentric smooth narrowing of the lumen of the pyloric part of stomach and proximal duodenum s/o benign stricture.

\section{Conclusion}

The spectrum of pathologies affecting stomach and duodenum in children are quite different from adults. US is the preferred screening modality; at the same time, radiographs and barium studies still play an important role in the characterization of these lesions. Knowledge of different abnormalities of stomach and duodenum and their clinical presentation help the radiologist in early detection and management of these conditions.

\section{Funding \\ None.}

\section{Conflict of Interest \\ None declared.}

\section{References}

1 Horjus Talabur Horje CS, Bruijnen R, Roovers L, Groenen MJM, Joosten FBM, Wahab PJ. Contrast enhanced abdominal ultrasound in the assessment of ileal inflammation in Crohn's disease: a comparison with MR enterography. PLoS One 2015;10(8):e0136105

2 Nievelstein RAJ, van Dam IM, van der Molen AJ. Multidetector CT in children: current concepts and dose reduction strategies. Pediatr Radiol 2010;40(8):1324-1344

3 Biassoni L, Easty M. Paediatric nuclear medicine imaging. $\mathrm{Br}$ Med Bull 2017;123(1):127-148

4 Lim RK, McKillop S, Karanicolas PJ, Scott L. Massive gastric pneumatosis from pyloric stenosis. CMAJ 2010;182(5):E227

5 Costa Dias S, Swinson S, Torrão $\mathrm{H}$, et al. Hypertrophic pyloric stenosis: tips and tricks for ultrasound diagnosis. Insights Imaging 2012;3(3):247-250

6 Currarino G. The value of double-contrast examination of the stomach with pressure "spots" in the diagnosis of infantile hypertrophic pyloric stenosis. Radiology 1964;83(5):873-878

7 Cohen HL, Zinn HL, Haller JO, Homel PJ, Stoane JM. Ultrasonography of pylorospasm: findings may simulate hypertrophic pyloric stenosis. J Ultrasound Med 1998;17(11): 705-711

8 Bawazir OA, Al-Salem AH. Congenital pyloric atresia: clinical features, diagnosis, associated anomalies, management and outcome. Ann Pediatr Surg 2017;13(4):188-193
9 Peterson CM, Anderson JS, Hara AK, Carenza JW, Menias CO. Volvulus of the gastrointestinal tract: appearances at multimodality imaging. Radiographics 2009;29(5):1281-1293

10 Singh JP, Rajdeo H, Bhuta K, Savino JA. Gastric Duplication Cyst: Two Case Reports and Review of the Literature. Available at: https://www.hindawi.com/journals/cris/2013/605059/. Accessed December 31, 2020

11 Zhang L, Chen Q, Gao Z, Xiong Q, Shu Q. Diagnosis and treatment of gastric duplication in children: A case report. Exp Ther Med 2017;14(4):3062-3066

12 Sigmon DF, Eovaldi BJ, Cohen HL, Duodenal Atresia and Stenosis. Treasure Island (FL): StatPearls Publishing; 2020

13 Erickson J, Retrouvey M, Rush J, Trace AP. Simultaneous duodenal stenosis and duodenal web in a newborn. Radiol Case Rep 2016;11(4):444-446

14 Mittal P, Gupta K, Mittal A, Gupta R. Imaging findings in incomplete annular pancreas in adults with crocodile jaw appearance: report of two cases. Int J Health Allied Sci 2016; 5(4):278-280

15 Applegate KE, Anderson JM, Klatte EC. Intestinal malrotation in children: a problem-solving approach to the upper gastrointestinal series. Radiographics 2006;26(5):1485-1500

16 Vilakazi M, Ismail F, Swanepoel HM, Muller EW, Lockhat ZI. Duodenal obstruction due to a preduodenal portal vein. Afr J Paediatr Surg 2014;11(4):359-361

17 Teele RL, Katz AJ, Goldman H, Kettell RM. Radiographic features of eosinophilic gastroenteritis (allergic gastroenteropathy) of childhood. AJR Am J Roentgenol 1979;132(4):575-580

18 Abuquteish D, Putra J. Upper gastrointestinal tract involvement of pediatric inflammatory bowel disease: a pathological review. World J Gastroenterol 2019;25(16):1928-1935

19 Khanna G, Kao SC, Kirby P, Sato Y. Imaging of chronic granulomatous disease in children. Radiographics 2005;25(5):1183-1195

20 Upadhyaya VD, Kumar B, Lal R. Sharma MS, Singh M, Rudramani. Primary duodenal tuberculosis presenting as gastric-outlet obstruction: its diagnosis. Afr J Paediatr Surg 2013; 10(2):83-86

21 Godara SC, George RA, Uniyal M. Case report: gastric tuberculosis - rare manifestation. Indian J Radiol Imaging 2004; 14(1):55-56

22 Amarapurkar DN, Patel ND, Amarapurkar AD. Primary gastric tuberculosis-report of 5 cases. BMC Gastroenterol 2003;3(1):6

23 Ghai S, Pattison J, Ghai S, O'Malley ME, Khalili K, Stephens M. Primary gastrointestinal lymphoma: spectrum of imaging findings with pathologic correlation. Radiographics 2007;27(5):1371-1388 
24 Quiroz HJ, Willobee BA, Sussman MS, et al. Pediatric gastrointestinal stromal tumors-a review of diagnostic modalities. Transl Gastroenterol Hepatol 2018;3:54

25 Lin C-H, Lin W-C, Lai I-H, Wu S-F, Wu K-H, Chen A-C. Pediatric gastric cancer presenting with massive ascites. World J Gastroenterol 2015;21(11):3409-3413

26 Valenzuela-Ramos MC, Mendizábal-Méndez AL, RíosContreras CA, Rodríguez-Montes CE. Pediatric gastric teratoma. J Radiol Case Rep 2010;4(10):6-13

27 Levy AD, Abbott RM, Rohrmann CA Jr, Frazier AA, Kende A. Gastrointestinal hemangiomas: imaging findings with pathologic correlation in pediatric and adult patients. AJR Am J Roentgenol 2001;177(5):1073-1081

28 Woodward K, Gangarosa LM, Hunt HV. Gastric inflammatory fibroid polyp. Indian J Pathol Microbiol 2011;54(3):622-623
29 Covarrubias DJ, Huprich JE; Armed Forces Institute of Pathology. Best cases from the AFIP. Juvenile polyposis of the stomach. Radiographics 2002;22(2):415-420

30 Ripollés T, García-Aguayo J, Martínez M-J, Gil P. Gastrointestinal bezoars: sonographic and CT characteristics. AJR Am J Roentgenol 2001;177(1):65-69

31 Sheybani EF, Gonzalez-Araiza G, Kousari YM, Hulett RL, MeniasCO.Pediatric nonaccidentalabdominaltrauma: what the radiologist should know. Radiographics 2014;34(1):139-153

32 Kamat R, Gupta P, Reddy YR, Kochhar S, Nagi B, Kochhar R. Corrosive injuries of the upper gastrointestinal tract: a pictorial review of the imaging features. Indian J Radiol Imaging 2019;29(1):6-13 